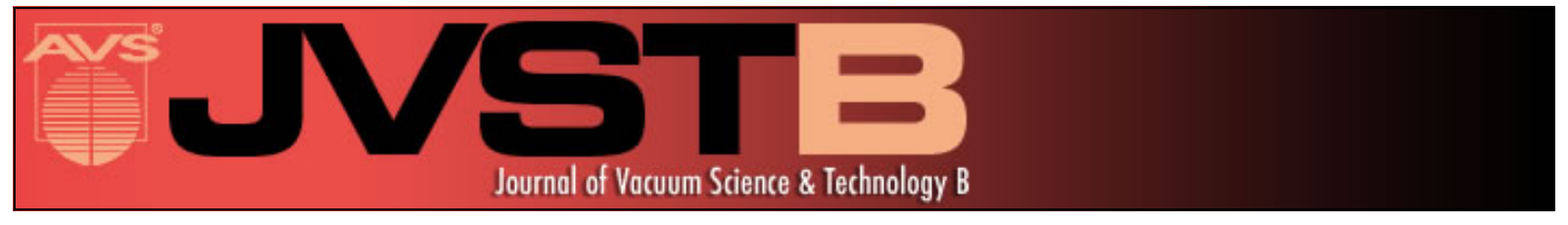

\title{
Towards a better understanding of the operative mechanisms underlying impurity-free disordering of GaAs: Effect of stress
}

Sachin Doshi, Prakash N. K. Deenapanray, H. H. Tan, and C. Jagadish

Citation: Journal of Vacuum Science \& Technology B 21, 198 (2003); doi: 10.1116/1.1535927

View online: http://dx.doi.org/10.1116/1.1535927

View Table of Contents: http://scitation.aip.org/content/avs/journal/jvstb/21/1?ver=pdfcov

Published by the AVS: Science \& Technology of Materials, Interfaces, and Processing

\section{Articles you may be interested in}

A model for arsenic anti-site incorporation in GaAs grown by hydride vapor phase epitaxy

J. Appl. Phys. 116, 243504 (2014); 10.1063/1.4904745

Arsenic antisite defects in $\mathrm{p}$-GaAs grown by metal-organic chemical-vapor deposition and the EL2 defect J. Appl. Phys. 106, 103704 (2009); 10.1063/1.3243162

Atomic relocation processes in impurity-free disordered $p-G a A s$ epilayers studied by deep level transient spectroscopy

Appl. Phys. Lett. 81, 3573 (2002); 10.1063/1.1519728

Impurity-free disordering mechanisms in GaAs-based structures using doped spin-on silica layers

Appl. Phys. Lett. 80, 4351 (2002); 10.1063/1.1484244

Enhanced As-Sb intermixing of GaSb monolayer superlattices in low-temperature grown GaAs

Appl. Phys. Lett. 79, 1294 (2001); 10.1063/1.1394166

\section{Instruments for Advanced Science}

Contact Hiden Analytical for further details: w www.HidenAnalytical.com E info@hiden.co.uk CLICK TO VIEW our product catalogue

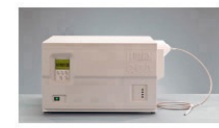

Gas Analysis

dynamic measurement of reaction gas streams catalysis and thermal analysis

molecular beam studies

fermentation, environmental and ecological studies

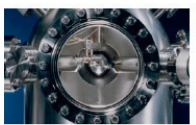

Surface Science UHVTPD

SIMS

end point detection in ion beam etch elemental imaging - surface mapping

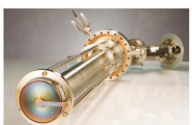

Plasma Diagnostics ; plasma source characterization etch and deposition process reaction kinetic studies analysis of neutral and radical species

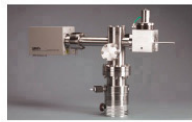

Vacuum Analysis - partial pressure measurement and control of process gases reactive sputter process control vacuum diagnostics vacuum coating process monitoring 


\title{
Towards a better understanding of the operative mechanisms underlying impurity-free disordering of GaAs: Effect of stress
}

\author{
Sachin Doshi, ${ }^{a), b)}$ Prakash N. K. Deenapanray, ${ }^{c}$ H. H. Tan, and C. Jagadish \\ Department of Electronic Materials Engineering, Research School of Physical Sciences and Engineering, \\ The Australian National University, Canberra ACT 0200, Australia
}

(Received 31 May 2002; accepted 11 November 2002; published 17 January 2003)

\begin{abstract}
The effect of stress on defect creation and diffusion during impurity-free disordering of $\mathrm{SiO}_{x}$-capped $n$-GaAs epitaxial layers has been investigated using deep level transient spectroscopy. The oxygen content in the $\mathrm{SiO}_{x}$ layer and the nature of the stress that it imposes on the GaAs layer were varied by changing the nitrous oxide flow rate, $N$, during plasma-enhanced chemical vapor deposition of the capping layer. The peak intensity of defects $S 1$ and $S 4$ increased with the increasing nitrous oxide flow rate to exhibit a maximum in the range $80 \mathrm{sccm}<N<200 \mathrm{sccm}$. Any further increase in $N$ resulted in a decrease in peak defect intensity, which reached an almost constant value for $N$ $>350 \mathrm{sccm}$. On the other hand, the peak intensity of $S 2 *$ increased linearly with $N$. We have explained the maximum in the intensity of defects $S 1$ and $S 4$ for $80 \mathrm{sccm}<N<200 \mathrm{sccm}$ to be due to a corresponding maximum in the compressive stress which is experienced by the capped GaAs layer during annealing. Although the creation of $S 2^{*}$, which we have proposed to be a complex involving the gallium vacancy $\left(V_{\mathrm{Ga}}\right)$, is enhanced with the increasing compressive stress, it also becomes efficiently converted into the arsenic-antisite, $\mathrm{As}_{\mathrm{Ga}}$. The compound effect of these opposing mechanisms results in a linear dependence of the peak intensity of $S 2 *$ on $N$. This study is to the best of our knowledge the first to provide the evidence for the stress-dependent anti-correlation between $V_{\mathrm{Ga}^{-}}$and $\mathrm{As}_{\mathrm{Ga}}$-related defects in GaAs. We have also narrowed the origin of $S 1$ to complexes involving arsenic interstitials, $\mathrm{As}_{i}$, and/or $\mathrm{As}_{\mathrm{Ga}}$. (C) 2003 American Vacuum
\end{abstract} Society. [DOI: 10.1116/1.1535927]

\section{INTRODUCTION}

Impurity-free disordering (IFD) using $\mathrm{SiO}_{2}$ capping layers has recently been used to modify the band gap of GaAsbased heterostructures to accomplish the monolithic integration of optoelectronic and photonic devices with different functionalities. ${ }^{1}$ The technological simplicity of this disordering process compared to current regrowth techniques lends itself as a desirable alternative to achieve band gap modification of compound semiconductor-based heterostructures.

Previous studies in the GaAs-based system have demonstrated that IFD is a complex process which is affected by several factors, including the type and quality of capping layers, capping layer deposition technique and annealing conditions. ${ }^{1-15}$ This dependence on multiple parameters and the current lack of understanding of the mechanisms that underlie the disordering process have precluded IFD from becoming a viable technology for the fabrication and integration of optoelectronic devices. Although IFD has been studied for at least two decades, only few studies have investigated the operative mechanisms of the disordering process. Defects play an important role in IFD, and a better understanding of the underlying defect engineering aspects may ultimately lead to an appreciation of the dominant operative

\footnotetext{
${ }^{a}$ Electronic mail: sdd109@rsphysse.anu.edu.au

${ }^{b}$ Also at: Faculty of Engineering and Information Technology, The Australian National University, Canberra ACT 0200, Australia.

${ }^{c}$ Author to whom correspondence should be addressed; electronic mail: pnk109@rsphysse.anu.edu.au
}

mechanisms of this process. There is the consensus that IFD proceeds via the diffusion of point defects on the group III sublattice in GaAs. However, defect creation and diffusion under conditions similar to those used for device fabrication and integration are not fully understood.

In an attempt to understand the defect engineering aspects of IFD, we have recently undertaken systematic defect studies on doped GaAs epilayers. ${ }^{16-19}$ Future studies will also include AlGaAs epilayers. It is worth noting here that defect studies in GaAs epilayers of the kind proposed in this study have become more pressing given the tremendous amount of research that is currently being pursued on $\mathrm{GaAs} /(\mathrm{In}) \mathrm{GaAsN}$ systems for the long-wavelength applications. ${ }^{20,21}$ We have recently demonstrated that three electron traps $S 1\left(E_{C}\right.$ $-0.23 \mathrm{eV}), S 2 *\left(E_{C}-0.53 \mathrm{eV}\right)$, and $S 4\left(E_{C}-0.74 \mathrm{eV}\right)$ are introduced in $\mathrm{SiO}_{2}$-capped MOCVD-grown $n$-type GaAs epilayers after rapid thermal annealing (RTA). We have proposed these defects to be complexes related to either gallium vacancies, $V_{\mathrm{Ga}}$, arsenic interstitials, $\mathrm{As}_{i}$, or arsenic antisites, $\mathrm{As}_{\mathrm{Ga}}$ (i.e., EL2-type defects) in GaAs. ${ }^{16,17}$ Another study has previously demonstrated that the stress that the dielectric capping layer imposes on the substrate plays during annealing plays an important role in the extent of disordering in GaAs-based heterostructures. ${ }^{5}$ In particular, that study proposed that IFD is a two-step mechanism, including the creation of $V_{\mathrm{Ga}}$ by the out-diffusion of $\mathrm{Ga}$ atoms into the capping layer, and the diffusion of $V_{\mathrm{Ga}}$ away from the capping layer-GaAs interface. The first step is energetically more favorable when the capping layer is under tensile stress, 
whereas the second step is enhanced when the near-surface region of GaAs is under compressive stress. ${ }^{5}$ However, the relative importance of the two steps is not known, mostly because the defect engineering aspects of IFD are not well understood. Furthermore, we have recently proposed that the efficient conversion of $V_{\mathrm{Ga}}$ into $\mathrm{As}_{\mathrm{Ga}}\left(V_{\mathrm{Ga}}+\mathrm{As}_{i} \rightarrow \mathrm{As}_{\mathrm{Ga}}\right)$, which influences the extent of IFD in GaAs/AlGaAs heterostructures, is also stress dependent. ${ }^{22}$

In this paper, we study the effect of stress on defect creation and diffusion in impurity-free disordered $n$-GaAs epilayers capped with $\mathrm{SiO}_{x}$ layers. We have changed both the oxygen content of the plasma-enhanced chemical vapor deposited $\mathrm{SiO}_{x}$ layer and the stress that it imposes onto the GaAs epilayer by varying the nitrous oxide flow rate, $N$. Our results can be adequately explained only after accounting for the effect of stress imposed by the $\mathrm{SiO}_{x}$ layers onto the $n$-GaAs epilayers. Defect depth profiles demonstrate the inverse relationship between the creation of $V_{\mathrm{Ga}}$ and $\mathrm{As}_{\mathrm{Ga}}$ in disordered samples, and a case is made for the efficient formation of $\mathrm{As}_{\mathrm{Ga}}$ under a compressive stress.

\section{EXPERIMENTAL PROCEDURE}

Epitaxial GaAs layers of (100) orientation and doped with $1 \times 10^{16} \mathrm{Si} \mathrm{cm}^{-3}$ were used. The $4 \mu \mathrm{m}$ thick epitaxial layers were grown by metalorganic chemical vapor deposition (MOCVD) on $n^{+}$-substrates. The epitaxial side of the samples were capped with $200 \mathrm{~nm} \mathrm{SiO}$ by plasma-enhanced chemical vapor deposition (PECVD) at constant rf power (20 $\mathrm{W})$, silane flow rate $(160 \mathrm{sccm})$, and substrate temperature $\left(295^{\circ} \mathrm{C}\right)$. The nitrous oxide flow rate, $N$, was varied between $40 \mathrm{sccm}$ and $700 \mathrm{sccm}$. We have previously reported on the comprehensive characterization of these $\mathrm{SiO}_{x}$ layers using Rutherford backscattering spectroscopy, spectroscopic ellipsometry, and Fourier transform infrared spectroscopy, as well as measuring the stress that the layers impose on the substrate on which they are deposited. ${ }^{6,7}$ Rapid thermal annealing (RTA) was performed on $\mathrm{SiO}_{x}$-capped samples at $900{ }^{\circ} \mathrm{C}$ for $30 \mathrm{~s}$ in an Ar atmosphere. After RTA, the capping layers were removed by chemical etching in $10 \%$ HF. Following a degreasing step, native oxide layers were removed by dipping the $\mathrm{GaAs}$ samples in dilute $\mathrm{HCl}$. Samples were loaded into a vacuum chamber to form circular Au Schottky barrier diodes (SBDs) by thermal evaporation at a base pressure of $5 \times 10^{-6}$ mbar. Deep level transient spectroscopy (DLTS) measurements were made using modified version of a lock-in based system. ${ }^{23}$ DLTS spectra were measured by adjusting bias conditions in order to probe the volume extending from $0.38 \mu \mathrm{m}$ to $0.74 \mu \mathrm{m}$ in all samples. DLTS depth profiles were measured a fixed reverse bias, $V_{r}$, and by successively incrementing the filling pulse, $V_{p}$, by $0.2 \mathrm{~V}$ up to zero bias conditions. The sample temperature was fixed at a value corresponding to the peak defect intensity in a given rate window. Furthermore, the defect depth profiles were calculated by accounting for the " $\lambda$-effect" according to Zohta and Watanabe. ${ }^{24}$ Current-voltage $(I-V)$ measurements were also performed on selected impurity-free disordered samples.

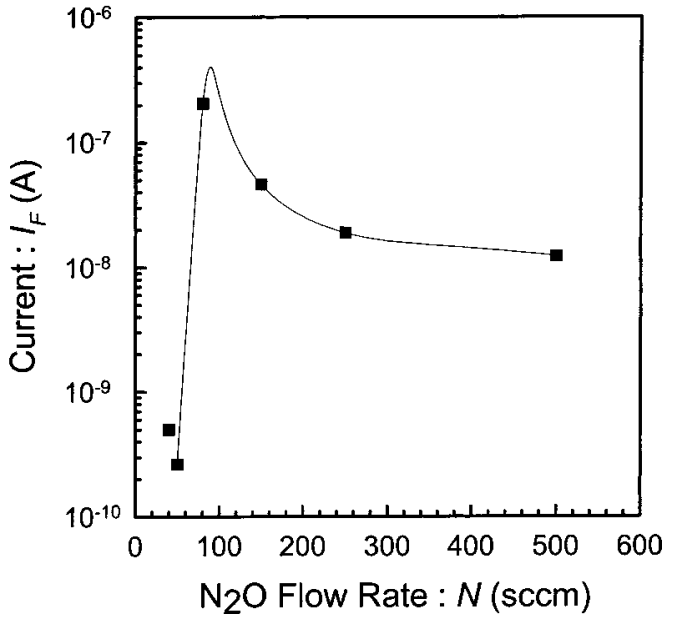

FIG. 1. Forward current, $I_{F}$, in various samples measured at a bias voltage of $0.1 \mathrm{~V}$ as a function of nitrous oxide flow rate, $N$. The solid line serves to guide the eyes only.

\section{RESULTS}

Since the zero bias depletion layer width in our samples is $\sim 0.32 \mu \mathrm{m}$, DLTS could not be used to probe the nearsurface region adjacent to the encapsulant/semiconductor $(E / S)$ interface. We have, therefore, performed $I-V$ measurements on the SBDs. Since the $I-V$ technique relies on the transfer of charge across the barrier between the semiconductor and metal contact, it exhibits extreme sensitivity to the surface modification of the GaAs epilayer. This is a key issue here since, as will be discussed later, the nearsurface region of GaAs is modified by the creation of defects in a region adjacent to the $E / H$ interface during IFD. The current measured in the various samples for a forward bias voltage of $0.1 \mathrm{~V}$ is plotted against the nitrous oxide flow rate, $N$, in Fig. 1. A small forward bias was chosen in order to measure the current transport predominantly due to carrier recombination in the space charge layer of disordered samples. ${ }^{25}$ It is observed that the forward current $I_{F}$ exhibits a maximum $\sim 10^{-7}$ A for samples capped with $\mathrm{SiO}_{x}$ layers for $80 \mathrm{sccm}<N<200 \mathrm{sccm}$. For the lower flow rates (i.e., $N<80 \mathrm{sccm}$ ), the current is significantly lower, while it levels off around $10^{-8} \mathrm{~A}$ for the higher flow rates.

DLTS was used to determine the concentrations and electronic properties of the defects introduced in the $n$-GaAs epilayers following disordering using $\mathrm{SiO}_{x}$ layers. The defect levels created in $\mathrm{SiO}_{x}$-capped samples are shown in DLTS spectra in Fig. 2(a) for selected nitrous oxide flow rates. The as-grown epilayer contained only the arsenic antisite related defect EL2, characteristic of MOCVD grown GaAs, in concentration $\sim 10^{12} \mathrm{~cm}^{-3} .{ }^{17}$ The reverse bias and filling pulse height were adjusted to probe the defects in the same volume of all the samples. IFD introduces the three dominant electron traps $S 1, S 2^{*}$, and $S 4 .{ }^{16-19}$ It can be seen from Fig. 2(a) that the relationship between the peak defect intensities and nitrous oxide flow rate is not straightforward. In order to highlight this nontrivial dependence, the peak defect intensities, $\Delta(d C / C)$, for $S 1, S 2^{*}$, and $S 4$ are plotted against $N$ in 

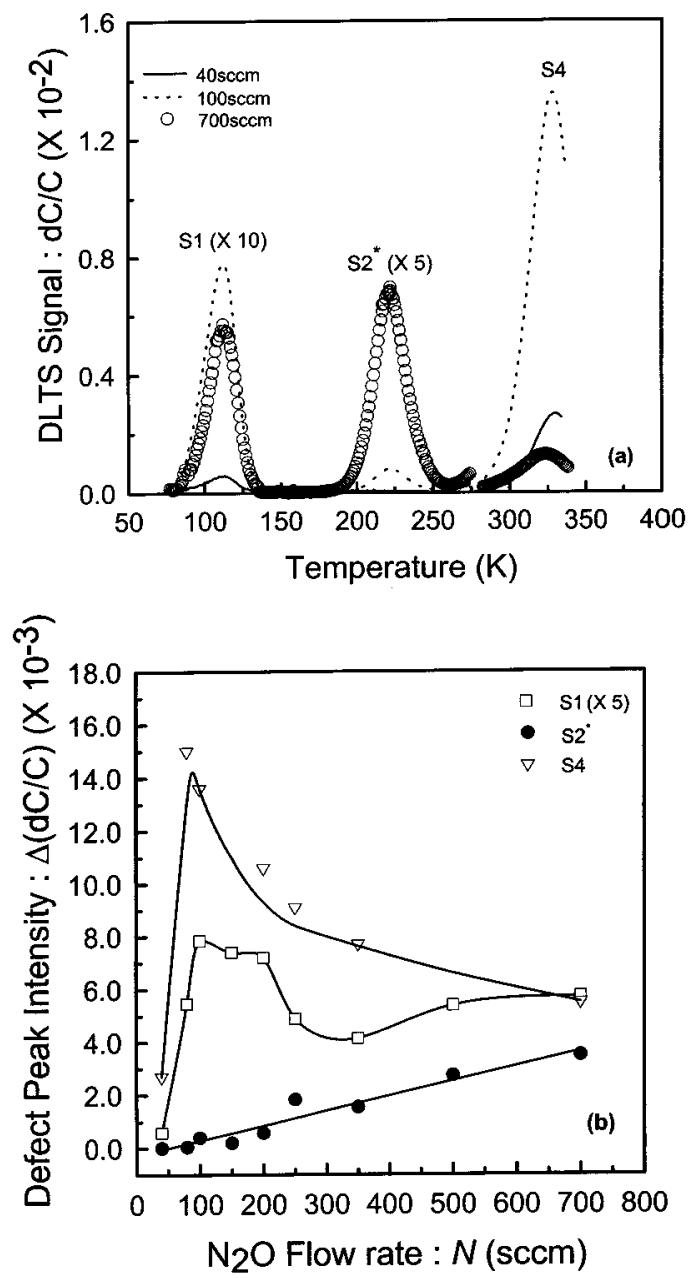

FIG. 2. (a) Typical DLTS spectra showing defects $S 1, S 2 *$, and $S 4$ in samples deposited at different nitrous oxide flow rate. The same volume was probed in all samples by adjusting the bias conditions, and (b) peak defect intensities versus nitrous oxide flow rate obtained from spectra in (a).

Fig. 2(b). It is clear that $S 1$ and $S 4$ demonstrate a fairly similar dependence on $N$, whereby the defect intensities initially increase with the increasing $N$ to reach a maximum in the flow rate regime $80 \mathrm{sccm}<N<200 \mathrm{sccm}$, and thereafter decrease to reach a constant level at the higher flow rates. The solid curves through the data points for $S 1$ and $S 4$ are only intended to guide the reader's eyes to highlight this trend. It is worth pointing out here that the flow rate dependence of $S 4$ in Fig. 2(b) is closely related to the results depicted in Fig. 1, and will be discussed later. The peak intensity of $S 2^{*}$, however, exhibits a different dependence on $N$, as it rather increases linearly with nitrous oxide flow rate. The results shown in Fig. 2 will be explained later in light of the oxygen content in $\mathrm{SiO}_{x}$ layers, together with the stress that they impose on the GaAs samples.

Successful defect engineering of the properties of semiconductors requires intimate knowledge of the "signatures" (i.e., activation energy, $E_{T}$, and capture cross section, $\sigma_{n}$ ), their concentrations and diffusion properties, amongst others, of defects introduced in the semiconductor material. We now show the concentration and diffusion properties of the de-
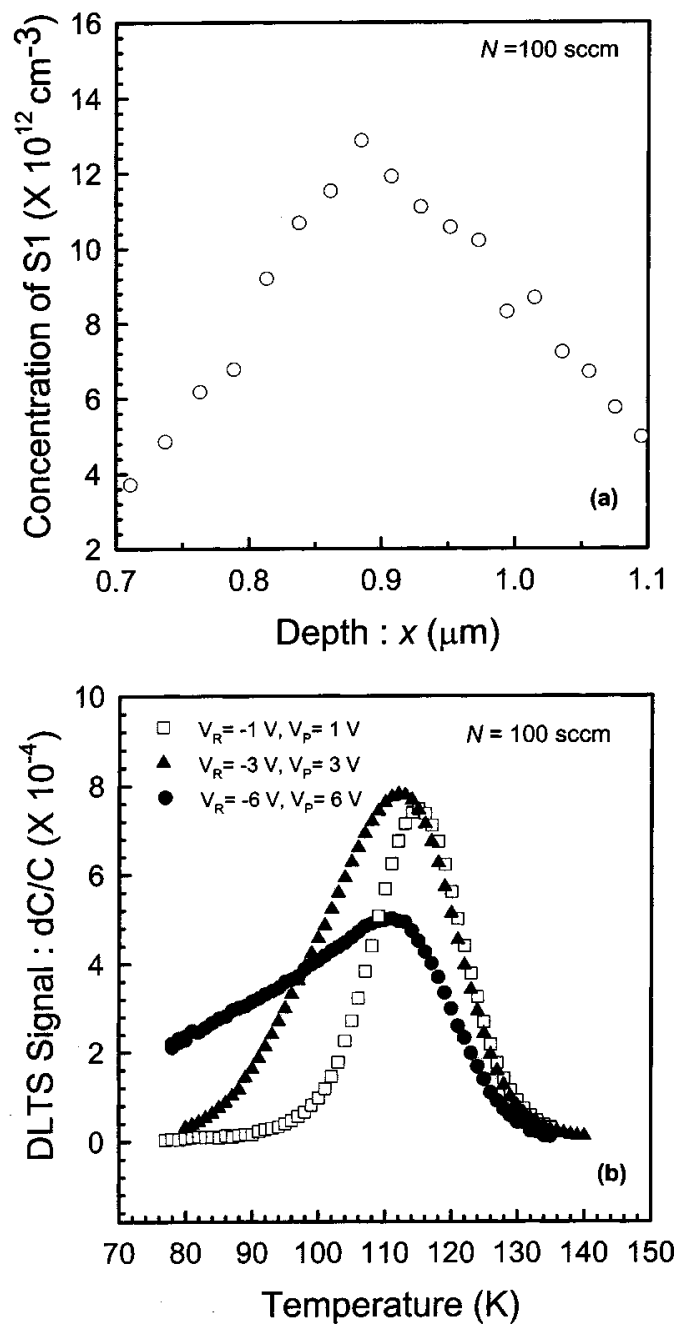

FIG. 3. (a) DLTS in-depth profile of $S 1$ for $N=100 \mathrm{sccm}$, and (b) DLTS spectra for defect $S 1$ obtained using the different bias conditions shown by legends.

fects by looking at their DLTS in-depth profiles. As evident from Fig. 2, the concentration of $S 1$ is relatively small for most values of $N$ except around $N=100 \mathrm{sccm}$. We, therefore, show the typical DLTS in-depth profile of $S 1$ for $N$ $=100 \mathrm{sccm}$ in Fig. 3(a), which is sufficient to illustrate one of its unique electronic properties. The concentration of $S 1$ exhibits an unusual parabolic dependence on depth with a conspicuous drop in the defect concentration towards the surface. The apparent decrease in the concentration of $S 1$ toward the surface can be explained by looking at the bias dependence of its DLTS peak shown in Fig. 3(b). Increasing the reverse bias causes the DLTS spectra to become skewed toward the lower temperatures. This profile broadening is accompanied by a shift in the defect peak position to the lower temperatures, as well as a decrease in the defect peak intensity. These results will be discussed in terms of the electric field-assisted electron emission characteristics of $S 1$ using supporting evidence from our previous results.

The typical DLTS depth profile of $S 2^{*}$ and $S 4$ are plotted in Fig. 4 for the selected nitrous oxide flow rate of $700 \mathrm{sccm}$. The measurements for $S 2 *$ were made using a filling pulse 


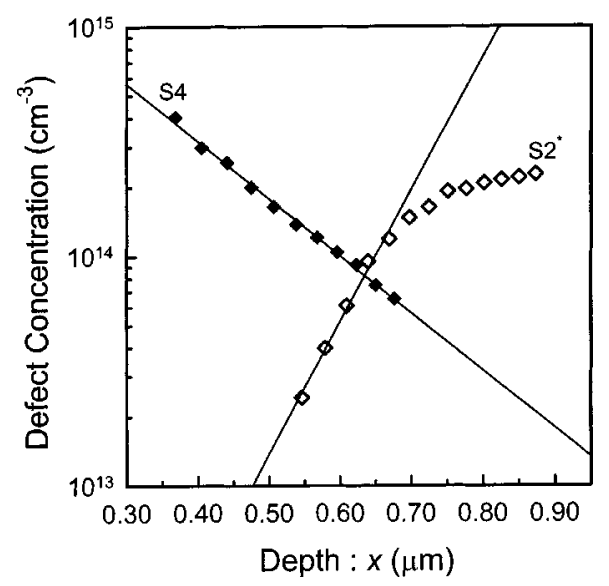

FIG. 4. Typical DLTS in-depth profiles of $S 2^{*}$ and $S 4$ for $N=700 \mathrm{sccm}$. A long filling pulse width $t_{p}=2 \mathrm{~s}$ was employed for the complete filling of the defect. The solid lines are least squares fits through the data points for $x$ $<\sim 0.7 \mu \mathrm{m}$, which reveal an exponential decrease in the concentration of $S 2 *$ toward the surface, and the exponential decay in the concentration of $S 4$ below the surface.

width, $t_{p}=2 \mathrm{~s}$. We have previously used variable pulse width measurements to show that the defect peak $S 2 *$ in Fig. 2(a) is in fact a superposition of two discrete defects. ${ }^{19}$ This accounts for the discrepancy between the smaller value of activation energy $\left(E_{C}-0.46 \mathrm{eV}\right)$ we have reported previously ${ }^{16,17}$ and the one reported in this study $\left(E_{C}\right.$ $-0.53 \mathrm{eV})$. We have provided a detailed explanation for this difference in an earlier study. ${ }^{18}$ A extremely small capture cross section $\left(\sim 10^{-20} \mathrm{~cm}^{2}\right)$ of the dominant of the two defects justifies the unusually long filling pulse width needed for the complete filling of $S 2 * .{ }^{19}$ The depth profile of $S 2 *$ exhibits the same features irrespective of the nitrous oxide flow rate. There is an exponential increase in the concentration of $S 2^{*}$ up to $\sim 0.7 \mu \mathrm{m}$, which then reaches a fairly constant level deeper in the samples. The least squares fit to the data points for depths less than $\sim 0.7 \mu \mathrm{m}$ reveals that the decay length of $S 2 *$ toward the surface is $\lambda_{1}=(0.08$ $\pm 0.02 \mu \mathrm{m})$. This characteristic decrease in the concentration of $S 2 *$ toward the surface is not related to the effect of electric field-assisted electron emission kinetics as is the case for $S 1$. It is better understood by considering the depth profile of $S 4$, as will be discussed shortly. The defect profile of $S 4$ in Fig. 4 clearly shows that it behaves quite differently to $S 2 *$. The defect concentration of $S 4$ decays exponentially with depth. The least squares fit is used to describe this behavior in the form $N_{S 4}=N_{T 0} \exp \left(-x / \lambda_{2}\right)$, where $\lambda_{2}$ is the characteristic decay length of $S 4$. The value of decay length for $S 4$ lies in the range $0.17 \mu \mathrm{m}<\lambda_{2}<0.19 \mu \mathrm{m}$. These results will now be discussed in light of the influence of stress on defect creation and diffusion during IFD. Despite that fact that IFD is a relatively simple technique, the results reported here demonstrate that the defect engineering underlying this process may not be straightforward.

\section{DISCUSSION}

The forward current, $I_{F}$, plotted in Fig. 1 shows that there is a peak diode current in the flow range $80 \mathrm{sccm}<N$
$<150 \mathrm{sccm}$. At room temperature, the dominant current transport mechanisms across the metal-semiconductor interface are through thermionic emission and recombination of electron-hole pairs in the space-charge region. The recombination of free carriers in the space-charge region normally takes place via localized states, and the most effective recombination centres are those lying near the middle of the band gap. ${ }^{25}$ Defects $S 1$ and $S 2 *$ being relatively nearer to the conduction band edge are thermally ionized at RT, and do not influence the free carrier transport process. It is, thus, reasonable to expect the forward current to follow the concentration of $S 4$, which influences carrier transport at and above room temperature. This is indeed the case as shown by the nitrous oxide flow rate dependence of the peak defect intensity of $S 4$ in Fig. 2(b).

We now turn to the variation of defect intensities with the nitrous oxide flow rate illustrated in Fig. 2(b). It is timely here to describe the two generally accepted mechanisms by which defects may be created in the impurity-free disordered GaAs epitaxial layers. ${ }^{1,5,6,7,12,13}$ First, an excess $V_{\mathrm{Ga}}$ may be created in the near-surface region of the GaAs due to the out-diffusion of $\mathrm{Ga}$ atoms into the $\mathrm{SiO}_{x}$ capping layer. The out-diffusion of $\mathrm{Ga}$ atoms into the dielectric layer is energetically favorable when the dielectric layer has a lower thermal expansion coefficient than GaAs. ${ }^{5}$ The out-diffusion of $\mathrm{Ga}$ atoms is also enhanced by the porosity of the capping layer. $^{2-4,10,15}$ Second, metallurgical reactions between the GaAs near-surface layer and the substoichiometric silicon oxide capping layer can also generate excess $V_{\mathrm{Ga}} \cdot{ }^{13}$ These two mechanisms for the creation of excess $V_{\mathrm{Ga}}$ suggest that disordering of GaAs should increase with the increasing oxygen content in the oxide capping layer. Indeed, a previous study has demonstrated that the concentration of $\mathrm{Ga}$ atoms out-diffusing from $\mathrm{GaAs}$ into a $\mathrm{SiO}_{x} N_{y}$ layer increased with the increasing oxygen content in the capping layer. ${ }^{2}$ Based on this argument alone disordering in our epilayers should have a direct correlation with the oxygen content in the $\mathrm{SiO}_{x}$ capping layers. In our recent studies, ${ }^{6,7}$ we have used RBS, FTIR, and spectroscopic ellipsometry to characterize the $\mathrm{SiO}_{x}$ capping layers deposited using different nitrous oxide flow rates. The oxygen content in the $\mathrm{SiO}_{x}$ layer increased monotonically with the increasing $N$ and near-stoichiometric films were obtained for $N \geqslant 350 \mathrm{sccm}$. It is worth noting that the intensity of $S 1$ and $S 4$ may well follow the variation of oxygen content in the $\mathrm{SiO}_{x}$ layer except for the maximum in the range $80 \mathrm{sccm}<N<200 \mathrm{sccm}$. On the other hand, $S 2 *$ increases linearly with $N$. Our results and discussion thus far have shown that although the increasing oxygen content results in larger defect concentrations, it cannot on its own fully explain the results in Figs. 1 and 2. We first discuss the results for $S 4$ and $S 2^{*}$, before concluding with $S 1$.

The maximum in the concentration of $S 4$ for $80 \mathrm{sccm}$ $<N<200 \mathrm{sccm}$ will now be explained in light of the stress that the capping layer imposes on the GaAs layer during RTA. In our previous studies, ${ }^{16,17}$ we demonstrated that $S 4$ was related to the antisite defect, $\mathrm{As}_{\mathrm{Ga}}$. Following the identification of EL2 as a complex involving $\mathrm{As}_{\mathrm{Ga}}$ and $\mathrm{As}_{i}$, 
whereby $\mathrm{As}_{i}$ is trapped in a second-neighbor position by the strain field of $\mathrm{As}_{\mathrm{Ga}},{ }^{26}$ the various second-neighbor positions result in a distribution of EL2 defects corresponding to slightly different energies. ${ }^{27}$ We have, therefore, argued that the lower activation energy of $S 4$ compared to EL2 in the as-grown MOCVD epilayer resulted from perturbations in the stain field of the defect due to the compressive stress imposed by the encapsulant onto the GaAs surface layer during IFD. These results are consistent with those of Ito et al. ${ }^{28}$ who showed that the formation of a similar defect in their samples was stress dependent. In Ref. 6 we have measured the stress that the $\mathrm{SiO}_{x}$ capping layer imposes on the substrate, and showed that the compressive stress on the GaAs layer exhibited a maximum in the flow regime $80 \mathrm{sccm}<N<250 \mathrm{sccm}$.

In light of the above argument, we, therefore, attribute the maximum in the peak intensity of $S 4$ for $80 \mathrm{sccm}<N$ $<200 \mathrm{sccm}$ to a corresponding maximum in the stress on the GaAs epilayers in this nitrous oxide flow rate regime. It is worth pointing out here that we have recently demonstrated the influence of stress on the formation of $S 4$ in $n$-GaAs epilayers using doped and undoped spin-on-glass (SOG) capping layers. ${ }^{22}$ We observed that the formation of $S 4$ was higher for the undoped SOG than for phosphorous-doped SOG since the former capping layer places the GaAs epilayer under higher compressive stress during annealing. The results in Fig. 4 demonstrate the exponential decay in the concentration of $S 4$ with characteristic length in the range $0.17 \mu \mathrm{m}<\lambda_{2}<0.19 \mu \mathrm{m}$. These values are consistent with the result we have reported earlier $(\sim 0.2 \mu \mathrm{m})$ when using a stoichiometric oxide layer (i.e., $\mathrm{SiO}_{2}$ ) for IFD. ${ }^{16}$ The exponential decrease in $S 4$ is characteristic of the diffusion of $\mathrm{As}_{i}$ from the near-surface region of the disordered GaAs. ${ }^{26,28}$

We now turn to the creation of $S 2 *$ and further relate its depth distribution to that of $S 4$. Following our previous results ${ }^{16-19}$ and those of Kuzuhara et al. ${ }^{29} S 2 *$ can be tentatively assigned as a defect complex involving $V_{\mathrm{Ga}}$ (i.e., $V_{\mathrm{Ga}}-\mathrm{Si}_{\mathrm{Ga}}$ or $\left.V_{\mathrm{Ga}}-\mathrm{As}_{\mathrm{Ga}}\right)$. In light of the influence of stress on the outdiffusion of $\mathrm{Ga}$ atoms into the capping layer, ${ }^{5}$ the creation of $V_{\mathrm{Ga}}$ should be a maximum for $80 \mathrm{sccm}<N$ $<200 \mathrm{sccm} .{ }^{6}$ Although this may be the case, the higher compressive stress also favors the efficient conversion of $V_{\mathrm{Ga}}$ into $\mathrm{As}_{\mathrm{Ga}}$. During IFD there is an increase in As:Ga ratio in the near-surface region of the GaAs layer due to the outdiffusion of Ga atoms into the capping layer. This results in the formation of excess $V_{\mathrm{Ga}}$ and $\mathrm{As}_{i}$, and ultimately the formation of $\mathrm{As}_{\mathrm{Ga}}$ (i.e., $V_{\mathrm{Ga}}+\mathrm{As}_{i} \rightarrow \mathrm{As}_{\mathrm{Ga}}$ ). The formation of $\mathrm{As}_{\mathrm{Ga}}$ is a precursor to the creation of $S 4$ through $\mathrm{As}_{\mathrm{Ga}}+\mathrm{As}_{i}$ $\rightarrow \mathrm{As}_{\mathrm{Ga}}-\mathrm{As}_{i}$. Consequently, we may anticipate a reduction in the concentration of $V_{\mathrm{Ga}}$ and, therefore $S 2^{*}$, when the formation of $S 4$ is enhanced. In other words, the increased introduction rate of $V_{\mathrm{Ga}}$ in the flow regime $80 \mathrm{sccm}<N$ $<200 \mathrm{sccm}$ is nullified by its efficient conversion into $\mathrm{As}_{\mathrm{Ga}}$. The compound result of these two opposing mechanisms is a linear variation of $S 2 *$ with the nitrous oxide flow rate. Further evidence of this is obtained by comparing the depth distributions of $S 2 *$ and $S 4$. The depth distributions of $S 2 *$ and $S 4$ are anti-correlated in the first $\sim 0.7 \mu \mathrm{m}$ below the GaAs surface. The different decay lengths for $S 2 *$ and $S 4$ in this region correspond to the diffusion of different species (i.e., $V_{\mathrm{Ga}}$ versus $\mathrm{As}_{i}$ ). Although we have recently proposed this model to explain the efficiency of Al-Ga interdiffsuion in impurity-free disordered $\mathrm{AlGaAs} / \mathrm{GaAs}$ using $\mathrm{SOG}^{22}$ the present study provides the experimental evidence for the efficient conversion of $V_{\mathrm{Ga}}$ into $\mathrm{As}_{\mathrm{Ga}}$ under a compressive stress in IFD GaAs epilayers. The fairly uniform concentration of $S 2 *$ for depths exceeding $\sim 0.7 \mu \mathrm{m}$ suggests that $V_{\mathrm{Ga}}$ may have a very long diffusion length, and that only those $V_{\mathrm{Ga}}$ contained in the compressed near-surface region are efficiently converted into $\mathrm{As}_{\mathrm{Ga}}$.

Although the exact nature of $S 1$ is unknown to us at present, we can, nevertheless, narrow down its origin based on the above discussions. As shown in Fig. 2(b), the peak defect intensity of $S 1$ shows a similar variation with $N$ as $S 4$. Hence, we may conclude that a compressive stress has a similar effect on the creation of $S 1$ as it has on $S 4$. We may, therefore, exclude $V_{\mathrm{Ga}}$ or complexes thereof as being the origin of $S 1$. In IFD of GaAs, the main defect components are $V_{\mathrm{Ga}}, \mathrm{As}_{i}$ and $\mathrm{As}_{\mathrm{Ga}}$, and their complexes. Hence, the results presented in this study suggest that the origin of $S 1$ may be narrowed down to $\mathrm{As}_{i}$ and/or $\mathrm{As}_{\mathrm{Ga}}$. The decrease in the concentration of $S 1$ toward the surface depicted in Fig. 3(a) should not be confounded to be similar to that of $S 2 *$. Figure 3(b) provides the evidence for this apparent decrease in the concentration of $S 1$ toward the surface. By varying the reverse bias which is applied to the Schottky diode, the magnitude of the electric field across the depletion region is increased. According to the depletion approximation, ${ }^{30}$ the electric field strength decreases from its maximum value of $\left(q N_{D} w / \varepsilon_{s}\right)$ [where $q, N_{D}, w$, and $\varepsilon_{s}$ are the electronic charge, doping concentration, depletion layer width, and dielectric constant of semiconductor, respectively] at the surface to zero at the edge of the depletion layer. For a fixed reverse bias (i.e., fixed depletion width, $w$ ), defects close to the surface experience a stronger field strength that those closer to the edge of the depletion layer. The DLTS spectra of $S 1$ shown in Fig. 3(b) unambiguously demonstrate that the ionization potential associated with the defect is significantly affected by an electric field. ${ }^{31}$ With the increasing reverse bias, and hence electric field strength within the depletion region, the ionization potential of $S 1$ is reduced, thereby requiring less thermal energy for electron emission to take place. This electric-field-assisted electron emission kinetics of $S 1$ translates into its DLTS spectrum to be skewed to the lower temperature. This produces a shift in the defect's peak position to the lower temperature, together with a decrease in its peak intensity. These results are consistent with our previous findings that the thermal activation energy of $S 1$ decreased with the increasing reverse bias applied to the diode. ${ }^{17}$ Hence, the apparent decrease in the concentration of $S 1$ toward the surface is a result of its electric-field-assisted electron emission characteristic. 


\section{CONCLUSION}

We have used deep level transient spectroscopy to characterize impurity-free disordered $n$-GaAs by employing oxide capping layers. The oxygen content in the plasmaenhanced chemical vapor deposited oxide layer was changed by varying the nitrous oxide flow rate. The peak defect intensity of $S 1$ and $S 4$ increased with the increasing nitrous flow rate to reach a maximum in the flow rate regime $80 \mathrm{sccm}<N<200 \mathrm{sccm}$, and thereafter decreased to reach an almost constant level. On the other hand, the peak defect intensity of $S 2 *$ increased linearly with the increasing nitrous oxide flow rate. We have demonstrated that the variation of oxygen content in the capping layer alone could not explain these results. The maximum in the peak intensity of $S 1$ or $S 4$ correspond to a maximum in the stress imposed on the GaAs layer by the capping layer for $80 \mathrm{sccm}<N$ $<200 \mathrm{sccm}$. This stress effect was expected to produce a similar effect on the creation of $S 2^{*}$, which we have proposed to be a complex involving $V_{\mathrm{Ga}}$. However, we have provided the evidence that the increase in compressive stress also favors the efficient conversion of $V_{\mathrm{Ga}}$ into $\mathrm{As}_{\mathrm{Ga}}$ through the defect reaction, $V_{\mathrm{Ga}}+\mathrm{As}_{i} \rightarrow \mathrm{As}_{\mathrm{Ga}}$. The increased production of $V_{\mathrm{Ga}}$ with an increasing compressive stress is nullified by its conversion into the arsenic antisite. Since $S 4$ is a member of the EL2 family of defects, i.e., a complex involving $\mathrm{As}_{\mathrm{Ga}}$, the depth profiles of $S 2 *$ and $S 4$ are anticorrelated within the first $\sim 0.7 \mu \mathrm{m}$ below the GaAs surface. Based on the results presented here, we have proposed that $S 1$ was most probably a complex involving $\mathrm{As}_{i}$ and/or $\mathrm{As}_{\mathrm{Ga}}$.

\section{ACKNOWLEDGMENTS}

P. N. K. Deenapanray and H. H. Tan gratefully acknowledge the financial support of the Australian Research Council.

${ }^{1}$ For a comprehensive review of impurity-free vacancy interdiffusion, see Semiconductor Quantum Wells Intermixing (Optoelectronic Properties of Semiconductors and Superlattices, Vol. 8), edited by E. H. Li (Gordon and Breach, Amsterdam, 2000), and Quantum Well Intermixing for Photonics, edited by E. H. Li (SPIE, Bellingham, 1997).

${ }^{2}$ S. Bürkner, M. Maier, E. C. Larkins, W. Rothermund, E. P. O’Reilly, and J. D. Ralston, J. Electron. Mater. 24, 805 (1995).

${ }^{3}$ P. N. K. Deenapanray, H. H. Tan, L. Fu, and C. Jagadish, Electrochem. Solid-State Lett. 3, 196 (2000).
${ }^{4}$ P. N. K. Deenapanray, H. H. Tan, M. I. Cohen, K. Gaff, M. Petravic, and C. Jagadish, J. Electrochem. Soc. 147, 1950 (2000).

${ }_{5}^{5}$ A. Pépin, C. Vieu, M. Schneider, H. Launois, and Y. Nissim, J. Vac. Sci. Technol. B 15, 142 (1997)

${ }^{6}$ P. N. K. Deenapanray and C. Jagadish, Electrochem. Solid-State Lett. 4 , G11 (2001).

${ }^{7}$ P. N. K. Deenapanray and C. Jagadish, J. Vac. Sci. Technol. B 19, 1962 (2001).

${ }^{8}$ P. Cusumano, B. S. Ooi, A. Saher Helmy, S. G. Ayling, A. C. Bryce, J. H. Marsh, B. Voegele, and M. J. Rose, J. Appl. Phys. 81, 2445 (1997)

${ }^{9}$ G. Li, S. J. Chua, S. J. Xu, X. C. Wang, A. Saher Helmy, Mao-Long Ke, and J. H. Marsh, Appl. Phys. Lett. 73, 3393 (1998).

${ }^{10}$ L. Fu, P. N. K. Deenapanray, H. H. Tan, C. Jagadish, L. V. Dao, and M. Gal, Appl. Phys. Lett. 76, 837 (2000).

${ }^{11}$ L. Fu, R. W. v. d. Heijden, H. H. Tan, C. Jagadish, L. V. Dao, and M. Gal, Appl. Phys. Lett. 80, 1171 (2002).

${ }^{12}$ A. Saher Helmy, J. S. Aitchison, and J. H. Marsh, Appl. Phys. Lett. 71, 2998 (1997).

${ }^{13}$ R. M. Cohen, G. Li, C. Jagadish, P. T. Burke, and M. Gal, Appl. Phys. Lett. 73, 803 (1998).

${ }^{14}$ E. V. K. Rao, A. Hamoudi, Ph. Krauz, M. Juhel, and H. Thibierge, Appl. Phys. Lett. 66, 472 (1995).

${ }^{15}$ A. Saher Helmy, S. K. Murad, A. C. Bryce, J. S. Aitchison, J. H. Marsh, S. E. Hicks, and C. D. W. Wilkinson, Appl. Phys. Lett. 74, 732 (1999).

${ }^{16}$ P. N. K. Deenapanray, H. H. Tan, C. Jagadish, and F. D. Auret, Appl. Phys. Lett. 77, 696 (2000).

${ }^{17}$ P. N. K. Deenapanray, H. H. Tan, C. Jagadish, and F. D. Auret, J. Appl. Phys. 88, 5255 (2000).

${ }^{18}$ P. N. K. Deenapanray, A. Martin, and C. Jagadish, Appl. Phys. Lett. 79, 2561 (2001).

${ }^{19}$ P. N. K. Deenapanray, M. Lay, D. Åberg, H. H. Tan, B. G. Svensson, F. D. Auret, and C. Jagadish, Physica B 308-310C, 776 (2002).

${ }^{20}$ M. Kondow, T. Kitatani, S. Nakatsuka, M. C. Larson, K. Nakahara, Y. Yazawa, and M. Okai, IEEE J. Sel. Top. Quantum Electron. 3, 719 (1997).

${ }^{21}$ L. H. Li, Z. Pan, Y. Q. Xu, Y. Du, Y. W. Lin, and R. H. Wu, Appl. Phys. Lett. 78, 2488 (2001).

${ }^{22}$ P. N. K. Deenapanray, Bin Gong, R. N. Lamb, A. Martin, L. Fu, H. H. Tan, and C. Jagadish, Appl. Phys. Lett. 80, 4351 (2002).

${ }^{23}$ B. G. Svensson, K.-H. Rydén, and B. M. S. Lewerentz, J. Appl. Phys. 66, 1699 (1989).

${ }^{24}$ Y. Zohta and M. O. Watanabe, J. Appl. Phys. 53, 1890 (1982).

${ }^{25}$ E. H. Rhoderick and R. H. Williams, in Metal-Semiconductor Contacts (Clarendon, Oxford, 1988), pp. 89-140.

${ }^{26}$ H. J. von Bardeleben, D. Stiévenard, D. Deresmes, A. Huber, and J. C. Bourgoin, Phys. Rev. B 34, 7192 (1986).

${ }^{27}$ T. Taniguchi and T. Ikoma, J. Appl. Phys. 54, 993 (1983).

${ }^{28}$ A. Ito, A. Kitagawa, Y. Tokuda, A. Usami, H. Kano, H. Noge, and T. Wada, Semicond. Sci. Technol. 4, 416 (1989).

${ }^{29}$ M. Kuzuhara, T. Nozaki, and T. Kamejima, J. Appl. Phys. 66, 5833 (1989).

${ }^{30}$ E. H. Rhoderick and R. H. Williams, in Metal-Semiconductor Contacts (Clarendon, Oxford, 1988), Appendix A.

${ }^{31}$ J. Frenkel, Phys. Rev. 54, 647 (1938). 